\title{
BEAM DYNAMICS PROBLEMS IN A MUON COLLIDER
}

\author{
Robert B. Palmer, Juan C. Gallardo, Richard C. Fernow, Harold Kirk, \\ I. Stumer, Y. Y. Lee, M. Syphers, Yağmur Torun ${ }^{\dagger}$ \\ David Winn $\ddagger \quad$ David Neuffer $\S$ \\ Yang Cho, James Norem 9 \\ Nikolai Mokhov, Robert Noble, Alvin Tollestrup " \\ Ronald Scanlan, Shlomo Caspi** Olivier Napoly ${ }^{\dagger \dagger}$
}

October 24, 2018

\begin{abstract}
We discuss the various beam dynamics problems in muon collider systems, starting from the proton accelerator needed to generate the muon beams and proceeding through the muon storage ring.
\end{abstract}

\footnotetext{
*E-mail:jcg@fel.cap.bnl.gov

${ }^{\dagger}$ Brookhaven National Laboratory, P. O. Box 5000, Upton, New York 11973-5000

${ }^{\ddagger}$ Fairfield University, Fairfield, CT, 06430-5195

$\S_{\text {CEBAF, Newport News, VA, } 23606}$

๑Argonne National Laboratory, Argonne, Illinois 60439-4815

"Fermi National Accelerator Laboratory, P.O. Box 500, Batavia, Illinois 60510

** Lawrence Berkeley Laboratory, Berkeley, CA 94720

${ }^{\dagger \dagger}$ DAPNIA-SEA, CE Saclay, 91191 Gif/Yvette CEDEX, France
} 


\section{INTRODUCTION}

Lepton $\left(e^{+} e^{-}\right)$colliders have the valuable property of producing simple, single-particle interactions with little background, and this property is essential in the exploration of new particle states. However, extension of $e^{+} e^{-}$ colliders to multi- $\mathrm{TeV}$ energies is severely performance-constrained by beamstrahlung, and cost-constrained because two full energy linacs are required [1]. On the other hand muons (heavy electrons) have negligible beamstrahlung, and can be accelerated and stored in rings.

The liabilities of $\mu$ 's are that they decay, with a lifetime of $2.2 \times 10^{-6} \mathrm{~s}$, and that they are created through decay into a diffuse phase space. In addition the decay products are likely to create large backgrounds at the final focus points making the detector design a challenge. The first problem is overcome by rapidly increasing the relativistic $\gamma$ factor; at $2 \mathrm{TeV}$ for example, the lifetime is $0.044 \mathrm{~s}$, sufficient for storage-ring collisions. The second can be dealt with by cooling. The possibility of muon colliders has been introduced by Skrinsky et al.[2], Neuffer [3], and others. More recently, several workshops and collaboration meetings have greatly increased the level of discussion [4], [5]. In this paper we discuss the beam dynamics problems encountered in one particular scenario for a $2+2 \mathrm{TeV}$ collider. Table 1 shows parameters for the candidate design. This scenario includes a high-intensity $\mu$-source, $\mu$-cooling, acceleration and storage in a collider. The complete cycle is repeated at $30 \mathrm{~Hz}$.

\section{SYSTEM COMPONENTS}

\section{Proton Driver}

The $\mu$-source driver is a high-intensity rapid-cycling $(30 \mathrm{~Hz})$ proton synchrotron. A recent study [6] suggests that an optimum proton energy may be $10 \mathrm{GeV}$. In this case we require a total of about $10^{14}$ protons at $30 \mathrm{~Hz}$. This

Table 1: Summary of Parameters of $2+2 \mathrm{TeV}$ Muon-Muon Collider

\begin{tabular}{|ll|c|}
\hline Beam energy & $\mathrm{TeV}$ & 2 \\
Beam $\gamma$ & $\mathrm{Hz}$ & 19,000 \\
Repetition rate & $10^{12}$ & 30 \\
Muons per bunch & & 2 \\
Bunches of each sign & 1 \\
Normalized rms emittance $\epsilon_{n}$ & $\mathrm{~mm}$ mrad & 50 \\
Average ring mag. field $B$ & Tesla & 6 \\
Effective turns before decay & & 900 \\
$\beta^{*}$ at intersection & $\mathrm{mm}^{-2} \mathrm{~s}^{-1}$ & 3 \\
Luminosity $\mathcal{L}$ & $\mathrm{cm}^{35}$ \\
\hline
\end{tabular}


specification is almost identical to that studied[7] at ANL for a spallation neutron source. The only difference is the number of bunches: 2 of $5 \times 10^{13}$ instead of one of $10^{14}$. One of which is for making $\mu^{-}$, the other for $\mu^{+}$, both brought onto the same target.

A problem specific to muon colliders is that the proton bunches should be short, with an rms bunch length less than $3 \mathrm{~ns}(1 \mathrm{~m})$.

An RF sequence must be designed to phase rotate the bunch prior to extraction. The total final momentum spread, based on the ANL parameters (95\% phase space of $4.5 \mathrm{Vs}$ per bunch), is modest ( $6 \%$, or $2.5 \% \mathrm{rms}$ ), but the space charge tune shift just before extraction would be very large $(\approx 1.5)$. A separate superconducting compression ring is thus needed (reducing the tune shift to $\approx 0.15$ ), or some other more exotic solution must be found. Some possible parameteres of the main components of the proton driver are given in table 2 .

Table 2: Proton Driver parameters

\begin{tabular}{|lll|c|}
\hline RFQ & Energy & $\mathrm{MeV}$ & 2 \\
& Frequency & $\mathrm{MHz}$ & 400 \\
\hline DTL & Energy & $\mathrm{MeV}$ & 68 \\
& Frequency & $\mathrm{MHz}$ & 400 \\
\hline Linac & Energy & $\mathrm{MeV}$ & 330 \\
& Gradient & $\mathrm{MeV} / \mathrm{m}$ & $4-5$ \\
& Frequency & $\mathrm{MHz}$ & 1200 \\
\hline Booster & Energy & $\mathrm{GeV}$ & 2.2 \\
& Circ. & $\mathrm{m}$ & 190 \\
& Frequency & $\mathrm{MHz}$ & $2.2-3$ \\
\hline Booster 2 & Energy & $\mathrm{GeV}$ & 10 \\
& Circ. & $\mathrm{m}$ & 690 \\
& Frequency & $\mathrm{MHz}$ & 9 \\
\hline Buncher & Energy & $\mathrm{GeV}$ & 10 \\
& Circ. & $\mathrm{m}$ & 70 \\
\hline Final & rms emittance & $\mathrm{mm} \mathrm{mrad}$ & 62 \\
& rms long. phase space & $\mathrm{V} \mathrm{s}$ & 0.7 \\
& rms bunch length & $\mathrm{ns}$ & 3 \\
& rms dp/p & $\%$ & 2.5 \\
\hline
\end{tabular}

\section{Target and Pion Capture}

The target could be either copper ( $24 \mathrm{~cm}$ by $12 \mathrm{~mm}$ diameter) or beryllium (70 $\mathrm{cm}$ by $2 \mathrm{~cm}$ diameter), although $\mathrm{Cu}$ would be preferred because of its higher pion multiplicity. Pions are captured from the target by a high-field hybrid solenoid that surrounds it. A field of 28 Tesla, and a radius of 7.5 $\mathrm{cm}$ are consistent with what is currently available [8]. The pions can then 
Table 3: Parameters of Phase Rotation Linacs

\begin{tabular}{|c|cccc|}
\hline Linac & $\begin{array}{c}\text { Length } \\
\mathrm{m}\end{array}$ & $\begin{array}{c}\text { Frequency } \\
\mathrm{MHz}\end{array}$ & $\begin{array}{c}\text { Gradient } \\
\mathrm{MeV} / \mathrm{m}\end{array}$ & $\begin{array}{c}\text { Phase } \\
\text { degrees }\end{array}$ \\
\hline 1 & 50 & 24 & 2 & 36 \\
2 & 50 & 24 & 2 & 0 \\
3 & 250 & 6 & 2 & 43 \\
4 & 60 & 24 & 2 & 81 \\
\hline
\end{tabular}

be matched, using a suitable tapered field [9] into a long $(350 \mathrm{~m})$ solenoidal decay channel. A field of 7 Tesla and radius of $15 \mathrm{~cm}$ for the decay channel seems reasonable and matches the capture acceptance.

Monte Carlo studies indicate that such a system captures almost $40 \%$ of the produced pions. Using the Wang [10] formula for pion production, the program calculates a yield of 0.22 muons, of each sign, per initial proton. However, for a $\mathrm{Cu}$ target, a higher multiplicity is expected and consequently, it would give a yet higher yield.

\section{Phase Rotation Linac}

The pions, captured by a solenoidal focusing system (and the muons into which pions decay) have a huge energy spread, from $0-3 \mathrm{GeV}$ (rms/mean $\approx 100 \%$ ), and would be difficult to transport and to handle in any subsequent system. It is thus proposed to introduce a linac along the decay channel, whose frequencies and phases are chosen to deaccelerate the fast particles and accelerate the slow ones; i.e. to phase rotate the muon bunch. Table 3 gives the parameters of these linacs.

After phase rotation the rms bunch length is $6 \mathrm{~m}$, and the rms momentum spread is reduced to about $15 \%$. Unfortunately, at such frequencies, the linacs cannot phase rotate both signs in the same bunch: hence the need for two bunches. The phases must be set to rotate the $\mu^{+}$'s of one bunch and the $\mu^{-}$'s of the other.

\section{Ionization Cooling}

\section{Cooling Theory}

For collider intensities, the phase-space volume must be reduced within the $\mu$ lifetime. Cooling by synchrotron radiation, conventional stochastic cooling and conventional electron cooling are all too slow. Optical stochastic cooling [11], electron cooling in an plasma discharge 12 and cooling in a crystal lattice 13] are being studied, but are not by any means certain. Ionization cooling of muons 14 seems relatively straightforward.

In ionization cooling, the beam loses both transverse and longitudinal momentum as it passing through a material medium. Subsequently, the 
longitudinal momentum can be restored by coherent reacceleration, leaving a net loss of transverse momentum. Ionization cooling is not practical for protons and electrons because of nuclear scattering (p's) and bremsstrahlung (e's) effects in the material, but is practical for $\mu$ 's because of their low nuclear cross section and relatively low bremsstrahlung.

The equation for transverse cooling (with energies in $\mathrm{GeV}$ ) is:

$$
\frac{d \epsilon_{n}}{d s}=-\frac{d E_{\mu}}{d s} \frac{\epsilon_{n}}{E_{\mu}}+\frac{\beta_{\perp}(0.014)^{2}}{2 E_{\mu} m_{\mu} L_{R}}
$$

where $\epsilon_{n}$ is the normalized emittance, $\beta_{\perp}$ is the betatron function at the absorber, $d E_{\mu} / d s$ is the energy loss, and $L_{R}$ is the material radiation length. The first term in this equation is the coherent cooling term and the second term is the heating due to multiple scattering. This heating term is minimized if $\beta_{\perp}$ is small (strong-focusing) and $L_{R}$ is large (a low-Z absorber).

From Eq.1 we find a limit to transverse cooling, which occurs when heating due to multiple scattering balances cooling due to energy loss. The limits are $\epsilon_{n} \approx 0.610^{-2} \beta_{\perp}$ for Li, and $\epsilon_{n} \approx 0.810^{-2} \beta_{\perp}$ for Be.

The equation for energy or longitudinal cooling is:

$$
\frac{d(\Delta E)^{2}}{d s}=-2 \frac{d\left(\frac{d E_{\mu}}{d s}\right)}{d E_{\mu}}<\left(\Delta E_{\mu}\right)^{2}>+\frac{d\left(\Delta E_{\mu}\right)_{\text {straggling }}^{2}}{d s}
$$

Where the first term is the cooling (or heating) due to energy loss and the second term is the heating due to straggling.

Cooling requires that $\frac{d\left(d E_{\mu} / d s\right)}{d E_{\mu}}>0$. But at energies below about $200 \mathrm{MeV}$, the energy loss function for muons, $d E_{\mu} / d s$, is rapidly decreasing with energy and there is thus rapid heating of the beam. Above $400 \mathrm{MeV}$ the energy loss function increases gently, thus giving some cooling, though not sufficient for our application.

In the long-path-length Gaussian-distribution limit, the heating term (energy straggling) is given by 15.

$$
\frac{d\left(\Delta E_{\mu}\right)_{\text {straggling }}^{2}}{d s}=4 \pi\left(r_{e} m_{e} c^{2}\right)^{2} N_{o} \frac{Z}{A} \rho \gamma^{2}\left(1-\frac{\beta^{2}}{2}\right)
$$

where $N_{o}$ is Avogadro's number and $\rho$ is the density. Since the energy straggling increases as $\gamma^{2}$, and the cooling system size scales as $\gamma$, cooling at low energies is desired.

Energy spread can also be reduced by artificially increasing $\frac{d\left(d E_{\mu} / d s\right)}{d E_{\mu}}$ by placing a transverse variation in absorber density at a location where position is energy dependent, i.e. where there is dispersion. The use of such wedges can reduce energy spread, but it simultaneously increases transverse emittance in the direction of the dispersion. Six dimensional phase space is not reduced. But it does allow the exchange of emittance between the energy and transverse directions. 


\section{Cooling System}

We require a reduction of the normalize transverse emittance by almost three orders of magnitude (from $2 \times 10^{-2}$ to $3 \times 10^{-5} \mathrm{~m}-\mathrm{rad}$ ), and a reduction of the longitudinal emittance by more than an order of magnitude. This cooling is obtained in a series of cooling cells. Each cell consists of a section of beryllium $(\approx 0.7 \mathrm{~m})$ or lithium $(\approx 2 \mathrm{~m})$ placed in a region of the lattice with a low $\beta_{\perp}$, a linac $(200 \mathrm{MeV})$, and a matching bend with dispersion where wedges can be introduced to interchange longitudinal and transverse emittance. The energy would be restricted to between 200 and $400 \mathrm{MeV}$, so as to avoid the energy $\mathrm{dE} / \mathrm{dx}$ heating below $200 \mathrm{MeV}$, but minimize the straggling heating at higher momenta. About 20 such cells would be needed.

For the early cells, when the emittance is still large, a sufficiently low $\beta_{\perp}$ can be obtained with solenoids. In later cells, when the emittance is lower and a lower $\beta_{\perp}$ is required, current carrying cooling rods (approx $2 \mathrm{~m}$ long, if $\mathrm{Li}$ ) which serve both to maintain the low $\beta_{\perp}$ and reduce the beam energy could be employed. In a lithium rod, with surface fields of 10 Tesla (as achieved in lithium lenses at Novosibirsk, FNAL and CERN [16]), a $\beta_{\perp}$ of $1.7 \mathrm{~cm}$ can be achieved, and the emittance is reduced to about $10^{-4} \mathrm{~m}$. But this is still a factor of $\approx 3$ above the emittance goal of Table 1 . A final stage might consist of short sections of Be at even lower $\beta_{\perp}$ insertions. Alternatively, the additional transverse emittance reduction can be obtained by cooling more than necessary longitudinally, and then exchanging transverse and longitudinal phase-space with a thick wedge absorber.

In all these cells lattices are required with adequate momentum acceptance, matching in and out of the low beta insertions, appropriate momentum compaction and control of emittance growth from space charge, wake field and resistive wall effects. In addition it would be desirable to economize on linac sections by forming groups of cells into recirculating loops.

\section{Acceleration}

Following cooling and initial bunch compression (to of the order of $0.2 \mathrm{~m}$ ) the beams must be accelerated to full energy (2 TeV). A single linac of this energy would work, but would be expensive, and would not utilize our ability to recirculate $\mu$ 's in rings. A conventional synchrotron cannot be used because the muons would decay before they were accelerated. A fast cycling synchrotron could be used but, because it would be limited to low magnetic fields, would be very large. The best solution seems to be a recirculating linac (similar to CEBAF). If acceleration is done in 20 recirculations, then only $100 \mathrm{GeV}$ of linear accelerator is required.

In practice, a cascade of at least 3 recirculating linacs (e.g., with maximum energies of $20 \mathrm{GeV}, 200 \mathrm{GeV}$ and $0.2 \mathrm{TeV}$ ) would be needed. The $\mu$-bunches would be compressed on each of the return arcs, and be bunched finally to the required length of $3 \mathrm{~mm}$ at full energy. The two higher energy recirculators must be superconducting for two reasons: the store time is far too long 
for conventional cavities, and the wall power consumption with conventional cavities would be too high. The total muon beam power is $38 \mathrm{MW}$. It is hoped to achieve at least $30 \%$ efficiency with superconducting cavities, giving a wall power consumption of $127 \mathrm{MW}$. The gradients assumed are below those assumed for TESLA. They may be over conservative in view of the shorter pulse duration in this application than assumed in TESLA. The muon linac beam dynamics is complicated by transverse HOM because of the large number of muons per bunch, about a factor of 100 higher than electrons in TESLA. The HOM power is estimated to be $\approx 100 \mathrm{~W} / \mathrm{m}$. As in the TESLA design, this would required a coupler section to remove this HOM power.

At the higher energies, space charge effects will not be a problem, but as the bunches are compressed wake field and resistive wall effects become serious. Preliminary studies suggest that, with a slight decrease in $Q / Z$ (by widening the irises, and with BNS damping, such effects can be controlled.

\section{$\mu$ Storage Ring}

After acceleration, the $\mu^{+}$and $\mu^{-}$bunches are injected into the $2-\mathrm{TeV}$ storage ring, with collisions in two low- $\beta^{*}$ interaction areas. The beam size at collision is $r=\sqrt{\epsilon_{n} \beta^{*}} \approx 2 \mu \mathrm{m}$, similar to hadron collider values. The bunch populations decay exponentially, yielding an integrated luminosity equal to its initial value multiplied by an "effective" number of turns $n_{\text {effective }}=150 \mathrm{~B}$, where $\mathrm{B}$ is the mean bending field in $\mathrm{T}$. With $9 \mathrm{~T}$ superconducting magnets, an average $\mathrm{B}$ of 6 Tesla might be obtained, yielding an $n_{\text {effective }} \approx 900$. The magnet design is complicated by the fact that the $\mu$ 's decay within the rings $\left(\mu \rightarrow e \nu_{e} \nu_{\mu}\right)$, producing electrons whose mean energy is approximately $1 / 3$ of that of the muons. These electrons travel to the inside of the ring dipoles, but radiate a substantial fraction of their energy, as synchrotron radiation, towards the outside of the ring. A warm tungsten liner of about 2 $\mathrm{cm}$ thickness will be required to intercept this radiation.

Even a preliminary study of resistive wall impedance instabilities indicate that $3 \mathrm{~mm}$ bunches of $2 \times 10^{12}$ muons would be unstable in a conventional ring. In any case, the rf requirements to maintain such a bunch are excessive. It is thus proposed to use an isochronous lattice of the type discussed by S.Y. Lee et al[17]. It remains to be seen if the required high degree of isochronism can in fact be achieved.

Another problem is the design of chromatic correction for the very low beta $\left(\beta^{*}=3 \mathrm{~mm}\right)$ insertions. A triplet design would have maximum beta's of $400 \mathrm{~km}$ in both directions, and chromaticity $\left(1 / 4 \pi \int \beta d k\right)$ of 3,700 in $\mathrm{x}$ and 3,500 in $y$. It seems clear that a local correction of chromaticity [18] would be required. A preliminary automated 19 study of such a correction system, using a doublet at the final focus, gave momentum acceptances of $\pm 0.1 \%$ and $\pm 0.6 \%$ in the two directions, where the $\beta_{\max }$ 's were 1.2 and 0.2 million $\mathrm{m}$ respectively. A similar design with the triplet $\left(\beta_{\max }\right.$ 's both 0.4 million $\mathrm{m}$ ) would be expected to give about $0.3 \%$ in both directions. More sophisticated designs [20] should do better. But this estimate is only for a 
Table 4: Detector Backgrounds from $\mu$ decay

\begin{tabular}{|l|cc|cc|}
\hline Location & \multicolumn{2}{|c|}{ outside } & \multicolumn{2}{c|}{ inside } \\
\hline & density & occupancy & density & occupancy \\
& $\mathrm{cm}^{-2}$ & $\%$ & $\mathrm{~cm}^{-2} \%$ & \\
\hline inner tracker & 170 & 0.07 & 480 & 0.19 \\
central tracker & 3.2 & 0.05 & 2.3 & 0.03 \\
outer tracker & 1.7 & - & 0.3 & - \\
\hline
\end{tabular}

single pass device like a linear collider; the performance for a storage ring remains to be seen.

\section{Detector Background}

For the physics user there is a problem of background from $\mu$-decays that occur through the whole collider ring, from near the interaction region and from scattering of any muon halo circulating in the ring.

A first Monte Carlo study 21] has been done with the MARS95 code 22, based on a preliminary insertion lattice. A contribution of direct decays has been studied in detail and a few ways to supress the background levels in a generic detector have been proposed. Also, from this study it is clear that much attention should be paid to muon halo contribution. A collimation system will be required in a straight section far from the detectors (presumably a quarter way around the ring). No such system has yet been designed.

Background track densities initiated by muon decays are indicated in Table 4 . In this study it was assumed that the detector pixels in the inner tracker were $20 \mu \mathrm{m}$ by $20 \mu \mathrm{m}$, and in the central tracker: $50 \mu \mathrm{m}$ by 300 $\mu m$. The track densities are high, but they result from very low energy electrons that would be eliminated in any track reconstruction. Given the fine subdivision of the assumed detector, the occupancies do not look impossible. However, it is hoped, and expected, that the background can be greatly reduced from these values by further improvements in shielding.

\section{CONCLUSION}

- The scenario for a $2+2 \mathrm{TeV}$, high luminosity collider is by no means complete. There are many problems still to be examined. Much work remains to be done, but:

- No obvious show stopper has yet been found.

- Many technical components require development: a large high field solenoid for capture, low frequency rf linacs, long lithium lenses, multibeam magnets for recirculation, warm bore shielding inside high field dipoles for the collider, muon collimators and background shields, but: 
- None of the required components may be described as exotic and their specifications are not far beyond what has been demonstrated.

- If the problems can be overcome, then a muon-muon colliders may be the best route to study physics at energies higher than those accessible at the LHC or NLC.

\section{ACKNOWLEDGMENTS}

We acknowledge extremely important contributions from our colleagues, especially C. Pellegrini, D. Cline, A. Chao, A. Ruggiero, A. Sessler, J. D. Bjorken, W. Barletta, D.Douglas, F. Mills, W. Willis, Dick Helms, Y. Zhao, H. Padamsee, John Irwin and Z. Parsa.

This research was supported by the U.S. Department of Energy under Contract No. DE-ACO2-76-CH00016 and DE-AC03-76SF00515.

\section{References}

[1] D. V. Neuffer, R. B. Palmer, Proc. European Particle Acc. Conf., London (1994); M. Tigner, in Advanced Accelerator Concepts, Port Jefferson, NY 1992, AIP Conf. Proc. 279, 1 (1993).

[2] E. A. Perevedentsev and A. N. Skrinsky, Proc. 12th Int. Conf. on High Energy Accelerators, F. T. Cole and R. Donaldson, eds., (1983)485; A. N. Skrinsky and V.V. Parkhomchuk, Sov. J. of Nucl. Physics 12, (1981) 3 .

[3] D. Neuffer, Colliding $\mu$ beams at 90 GeV, Fermi Lab Note, FN-319, July (1979); D. Neuffer, Particle Accelerators 14 (1983) 75; D. Neuffer, Proc. 12th Int. Conference on High Energy Accelerators, (1983) 481.

[4] Proceedings of the Mini-Workshop on $\mu^{+} \mu^{-}$Colliders: Particle Physics and Design, Napa CA, to be published in Nucl Inst. and Meth. A (1993); Proceedings of the Muon Collider Workshop, February 22, 1993, Los Alamos National Laboratory Report LA- UR-93-866 (1993) and $2^{\text {nd }}$ Workshop on Physics Potential $\&$ Development of $\mu^{+} \mu^{-}$Colliders, Sausalito, CA (1994).

[5] Transparencies at the $2+2 \mathrm{TeV} \mu^{+}-\mu^{-}$Collider Collaboration Meeting, Feb 6-8, 1995, BNL, compiled by Juan C. Gallardo.

[6] R. B. Palmer et.al., Monte Carlo Simulations of Muon Production, submitted to the Sausalito Proceedings.

[7] Y. Cho, et. al., ANL-PUB-081622, Proc. Int. Collab. on Advanced Neutron Sources, Abbingdon, UK., May 24-28 (1993). 
[8] Physics Today, Dec (1994), p21-22

[9] R. Chehab, J. Math. Phys. 5, (1978) 19

[10] C. L. Wang, Phys. Rev. D10, (1974) 3876; C. L. Wang, Phys Rev D9, (1973) 2609 and Phys. Rev. D10, (1974) 3876.

[11] A. A. Mikhailichenko and M. S. Zolotorev, Phys. Rev. Lett. 71, (1993) 4146; M. S. Zolotorev and A. A. Zholents, SLAC-PUB-6476 (1994)

[12] Ady Hershcovitch, Brookhaven National Report AGS/AD/Tech. Note No. 413 (1995)

[13] Z. Huang, P. Chen, R. Ruth, SLAC-PUB-6745, Proc. Workshop on Advanced Accelerator Concepts, Lake Geneva, WI, June (1994); P. Sandler presentation at Sausalito Workshop, to be published; A. Bogacz, private communication.

[14] Initial speculations on ionization cooling have been variously attributed to G. O'Neill and/or G. Budker see D. Neuffer, Particle Accelerators, 14, (1983) 75; D. Neuffer, Proc. 12th Int. Conf. on High Energy Accelerators, F. T. Cole and R. Donaldson, eds., 481 (1983); D. Neuffer, in Advanced Accelerator Concepts, AIP Conf. Proc. 156, 201 (1987); E. A. Perevedentsev and A. N. Skrinsky, Proc. 12th Int. Conf. on High Energy Accelerators, F. T. Cole and R. Donaldson, eds., 485 (1983); A. N. Skrinsky and V.V. Parkhomchuk, Sov. J. of Nucl. Physics 12, (1981) 3.

[15] U. Fano, Ann. Rev. Nucl. Sci. 13, 1 (1963).

[16] G. Silvestrov, Proceedings of the Muon Collider Workshop, February 22, 1993, Los Alamos National Laboratory Report LA-UR-93-866 (1993); B. Bayanov, J. Petrov, G. Silvestrov, J. MacLachlan, and G. Nicholls, Nucl. Inst. and Meth. 190, (1981) 9; Colin D. Johnson, Hyperfine Interactions, 44 (1988) 21; M. D. Church and J. P. Marriner, Annu. Rev. Nucl. Sci. 43 (1993) 253.

[17] S.Y. Lee, K. Y. Ng, D. Trbojevic, FNAL Report FN595 (1992); Phys. Rev. E48, (1993) 3040.

[18] K. L. Brown, J Spencer, SLAC-PUB-2678 (1981) presented at the Particle Accelerator Conf., Washington, (1981) and K.L. Brown, SLAC-PUB4811 (1988), Proc. Capri Workshop, June 1988 and J.J. Murray, K. L. Brown, T.H. Fieguth, Particle Accelerator Conf., Washington, 1987.

[19] Bruce Dunham, Olivier Napoly, FFADA, Final Focus. Automatic Design and Analysis, CERN Report CLIC Note 222, (1994); Olivier Napoli, it CLIC Final Focus System: Upgraded Version with Increased Bandwidth ans Error Analysis, CERN Report CLIC Note 227, (1994). 
[20] Oide, SLAC-PUB-4953 (1989); J. Irwin, SLAC-PUB-6197 and LBL33276, Particle Accerator Conf.,Washington, DC, May (1993); R. Brinkmann, Optimization of a Final Focus System for Large Momentum Bandwidth, DESY-M-90/14 (1990).

[21] G. W. Foster, and N. V. Mokhov, Backgrounds and Detector Performance at $2+2 \mathrm{TeV} \mu^{+} \mu^{-}$Collider, Fermilab-Conf-95/037 (1995); to be published by AIP in the Proceedings of the Sausalito Workshop.

[22] N. V. Mokhov, The MARS10 Code System, Fermilab FN-509 (1989); The MARS95 Code System (User's Guide), to be published as Fermilab Report. 